\title{
A novel microduplication of ARID1B: Clinical, genetic, and proteomic findings
}

\author{
Catarina M. Seabra ${ }^{1,2,3}$ | Nicholas Szoko ${ }^{4}$ | Serkan Erdin ${ }^{2,3}$ \\ Ashok Ragavendran $^{2,3}$ | Alexei Stortchevoi ${ }^{2}$ | Patrícia Maciel ${ }^{5,6}$ | \\ Kathleen Lundberg ${ }^{7}$ | Daniela Schlatzer ${ }^{7}$ | Janice Smith ${ }^{8}$ | \\ Michael E. Talkowski ${ }^{2,3,9}$ | James F. Gusella ${ }^{2,3,10}$ | Marvin R. Natowicz ${ }^{4,11,12}$ (i) \\ ${ }^{1}$ GABBA - Institute of Biomedical Sciences Abel Salazar of the University of Porto, Porto, Portugal \\ 2 Molecular Neurogenetics Unit, Center for Genomic Medicine, Massachusetts General Hospital, Boston, Massachusetts \\ 3 Program in Medical and Population Genetics, Broad Institute of MIT and Harvard Medical School, Boston, Massachusetts \\ ${ }^{4}$ Cleveland Clinic Lerner College of Medicine, Cleveland, Ohio \\ ${ }^{5}$ Life and Health Sciences Research Institute, School of Medicine, University of Minho, Braga, Portugal \\ ${ }^{6}$ ICVS/3Bs - PT Government Associate Laboratory, Braga/Guimarães, Portugal \\ ${ }^{7}$ Center for Proteomics, Case Western Reserve University School of Medicine, Cleveland, Ohio \\ ${ }^{8}$ Baylor Genetics Laboratories, Baylor College of Medicine, Houston, Texas \\ 9 Department of Neurology, Harvard Medical School, Harvard University, Boston, Massachusetts \\ 10 Department of Genetics, Harvard Medical School, Harvard University, Boston, Massachusetts \\ 11 Pathology \& Laboratory Medicine, Genomic Medicine, Neurology and Pediatrics Institutes, Cleveland Clinic, Cleveland, Ohio \\ 12 Department of Pathology, Case Western Reserve University School of Medicine, Cleveland, Ohio
}

\section{Correspondence}

Marvin R. Natowicz, Cleveland Clinic Lerner

College of Medicine, Cleveland Clinic, Mail Code LL3, 9500 Euclid Avenue, Cleveland 44195, OH. Email: natowim@ccf.org

Funding information

Simons Foundation Autism Research Initiative, Grant numbers: 308955, ROOMH095867; Autism Research Institute; NIH, Grant number: GM061354; FCT fellowship, Grant number:

FCT fellowship SFRH/BD/5209/2012
Genetic alterations of ARID1B have been recently recognized as one of the most common mendelian causes of intellectual disability and are associated with both syndromic and non-syndromic phenotypes. The ARID1B protein, a subunit of the chromatin remodeling complex SWI/SNF-A, is involved in the regulation of transcription and multiple downstream cellular processes. We report here the clinical, genetic, and proteomic phenotypes of an individual with a unique apparent de novo mutation of $A R I D 1 B$ due to an intragenic duplication. His neurodevelopmental phenotype includes a severe speech/language disorder with full scale IQ scores 7898 and scattered academic skill levels, expanding the phenotypic spectrum of ARID1B mutations. Haploinsufficiency of ARID1B was determined both by RNA sequencing and quantitative RT-PCR. Fluorescence in situ hybridization analysis supported an intragenic localization of the ARID1B copy number gain. Principal component analysis revealed marked differentiation of the subject's lymphoblast proteome from that of controls. Of 3426 proteins quantified, 1014 were significantly up- or down-regulated compared to controls $(q<0.01)$. Pathway analysis revealed highly significant enrichment for canonical pathways of EIF2 and EIF4 signaling, protein ubiquitination, tRNA charging and chromosomal replication, among others. Network analyses revealed down-regulation of: (1) intracellular components involved in organization of membranes, organelles, and vesicles; (2) aspects of cell cycle control, signal 
transduction, and nuclear protein export; (3) ubiquitination and proteosomal function; and (4) aspects of mRNA synthesis/splicing. Further studies are needed to determine the detailed molecular and cellular mechanisms by which constitutional haploinsufficiency of $A R I D 1 B$ causes syndromic and non-syndromic developmental disabilities.

\section{KEYWORDS}

ARID1B, chromatin, development, intellectual disability, proteome, proteomic, regulation, SWI/SNF, SWI/SNF-A

\section{1 | INTRODUCTION}

Intellectual disability is characterized by significant limitations in cognitive functioning and adaptive behaviors (American Psychiatric Association, 2013) and affects $1-3 \%$ of the general population. Mutations of ARID1B (AT-rich interactive domain 1B) are an epidemiologically significant subset of mendelian causes of neurodevelopmental disability and are associated with non-syndromic intellectual disability as well as syndromic forms of intellectual disability such as Coffin-Siris syndrome (Hoyer et al., 2012; Santen \& Clayton-Smith, 2014; Sim, White, \& Lockhart, 2015). The product of $A R I D 1 B$ is a ubiquitous nuclear-localized protein that is a subunit of SWI/SNF-A, a chromatin remodeling complex that contains over 25 core subunits and that is involved in the regulation of many biological processes, including regulation of transcription (Euskirchen, Auerbach, \& Snyder, 2012). ARID1B mutations associated with intellectual disability include whole gene deletions, intragenic deletions, splice site, nonsense, and frameshift mutations, all of which point to haploinsufficiency as the mechanism causing the phenotype, as well as rare and less wellstudied duplications (reviewed in Santen \& Clayton-Smith, 2014 Sim et al., 2015). Here, we report the clinical, genetic, and proteomic findings of an individual having a unique loss-offunction mutation of $A R I D 1 B$ due to an intragenic duplication.

\section{2 | CLINICAL REPORT}

This study was approved by the Institutional Review Board of the Cleveland Clinic. The subject is a 14-year-old male born at 39 weeks of gestation to a healthy primagravida 38-year-old mother and unrelated 49-year-old father with non-contributory family histories. There were no medical concerns during infancy. Early developmental milestones were met until 1-year of age but no use of sentences occurred until about $2.5-3$ years old.

Physical examinations during early childhood noted language delays, borderline macrocephaly, strabismus, dysarthria, mild hypotonia and mild gross and fine motor incoordination. Diminished physical endurance was also apparent in early childhood and has persisted. He had prolonged recovery times from illnesses, including several developmental regressions that lasted 2 or more months between ages 7-9 years, as well as two episodes of difficulty recovering from general anesthesia at 3 and 6 years old. Ophthalmologic exam revealed a right optic nerve pit. Growth parameters at 6.5 years included head circumference $54.8 \mathrm{~cm}$ (98\%), weight $22.1 \mathrm{~kg}$ (52\%), and height $116.9 \mathrm{~cm}$ (38\%). Physical exam at 12.4 years showed a non-dysmorphic male with weight $36.6 \mathrm{~kg}(18 \%)$ and height $140.3 \mathrm{~cm}$ (7\%); neurological exam showed slowed processing to questions or directions, abnormal gait with bilateral intoeing, mild imbalance, mild dysmetria, slow rapid alternating movements, and clumsy fine motor function.

The earliest neuropsychological assessment, at 3.5 years, used the Stanford Binet 5 tool and showed full-scale IQ 98, verbal IQ 122, and non-verbal IQ 74. At 5 years, using the WPPSI-III, he was noted to have a full-scale IQ 83, verbal IQ 78, and non-verbal IQ 96, a poor fund of general knowledge and difficulty formulating and expressing verbal concepts. At 8 years, using the WISC-4, there was a full-scale IQ 78, with verbal comprehension 85 , perceptual reasoning 102 , working memory 86 , and processing speed 50 . His strongest skills related to nonverbal visual-spatial reasoning. There was slow processing of information, deficiency of working memory, and poor visual/graphomotor skills. He was diagnosed with a severe language disorder. Auditory evaluation at 10 years showed an auditory processing disorder with severe difficulty in figure-ground discrimination, integration of words and sentences, temporal integration and phonological processing, and low average auditory comprehension, and average auditory short-term memory. At 12.5 years, using the Wide Range Achievement Test-4, he scored 101, 104,91 , and 67 in word reading, sentence comprehension, spelling, and math computation, respectively.

Cranial MRIs at 6 months and 7 years of age showed a mildly dysmorphic corpus callosum. A $48 \mathrm{hr}$ EEG at 5 years was unremarkable. Routine blood tests and urinalysis were normal. Normal metabolic testing included thyroid function tests, plasma amino acids, blood ammonia, and urinary organic acids, acylglycines, guanidinoacetate and creatine. Newborn screening, including for galactosemia, was negative. A fasting global plasma metabolomic analysis was unremarkable. The blood lactate and plasma butyrylcarnitine levels were intermittently increased. The fibroblast lactate:pyruvate ratio was normal, as were activities of 
fibroblast electron transport chain complexes II, III, and IV and pyruvate dehydrogenase. A fibroblast loading study for defects of mitochondrial fatty acid beta-oxidation was negative. A lymphocyte cytogenetic analysis showed a normal 46,XY karyotype at the 550 band resolution. Whole exome sequencing did not reveal pathogenic variants that were likely or definitely related to the clinical phenotype. A separate sequencing of the mitochondrial genome was unremarkable.

Array CGH showed a copy number gain within chromosome band 6q25.3 of approximately $0.361 \mathrm{Mb}$ in size, arr [GRCh37] 6q25.3 (157133792-157495187)x3 dn (Figure 1b). The duplication, which was not observed in either parent, involves a segment containing exons 2-10 (ENST00000346085) of ARID1B. Array analysis also showed heterozygosity for an approximately $0.003 \mathrm{Mb}$ maternally inherited copy number loss, arr [GRCh37] 9p13.3(3464759834650608)x1 mat, including part of GALT. There was no clinical or biochemical evidence for galactosemia, nor was a mutation of the other GALT allele detected.

\section{3 | MATERIALS AND METHODS}

\subsection{Molecular cytogenomic analysis}

Array $\mathrm{CGH}$ was performed on a $400 \mathrm{~K}$ oligonucleotide microarray (version 10.2) designed by the Medical Genetics Laboratories at Baylor College of Medicine and manufactured by Agilent Technologies (Santa Clara, CA). It includes exonic coverage of over 4,000 candidate and disease genes at an average resolution of $30 \mathrm{~kb}$ with 60,000 SNP probes and 670 probes for the mitochondrial genome. Data was extracted using Agilent's Feature Extraction software (version 9.5.3.1) and was analyzed using a web-based software platform (Cheung et al., 2005; Lu et al., 2007).

\section{2 | Transcriptome analysis}

Gene expression was measured by RNAseq and quantitative RT-PCR. Total RNA was extracted from a patient-derived EBV-transformed lymphoblastoid cell line (LCL) and control EBVtransformed LCLs using TRIzol ${ }^{\circledR}$ (Invitrogen, Carlsbad, CA) followed by RNeasy Mini Kit (Qiagen, Hilden, Germany) column purification. cDNA was synthetized from $1 \mu \mathrm{g}$ of extracted RNA using SuperScript ${ }^{\circledR}$ II Reverse Transcriptase (ThermoFisher Scientific, Waltham, MA) with oligo(dT), random hexamers, and RNase inhibitor. The RNAseq library was prepared using the Illumina TruSeq kit and manufacturer's instructions (Illumina, San Diego CA). Libraries were multiplexed, pooled and sequenced on multiple lanes of an Illumina HiSeq2500, generating an average of 30 million paired-end reads of $76 \mathrm{bp}$. Quality assessment of sequence reads was performed using fastQC (v. 0.10.1) (http://www. bioinformatics.babraham.ac.uk/projects/fastqc/). Sequence reads were then aligned to human reference genome Ensembl GRCh37 (v. 71) using GSNAP (v. 12-19-2014) (Wu \& Nacu, 2010) at its default parameter setting. Quality checking of alignments was assessed by a custom script utilizing Picard Tools (http:// broadinstitute.github.io/picard/), RNASeQC (DeLuca et al., 2012), RSeQC (Wang, Wang, \& Li, 2012), and SamTools (Li et al., 2009). Novel transcript analysis was performed using Cufflinks, and

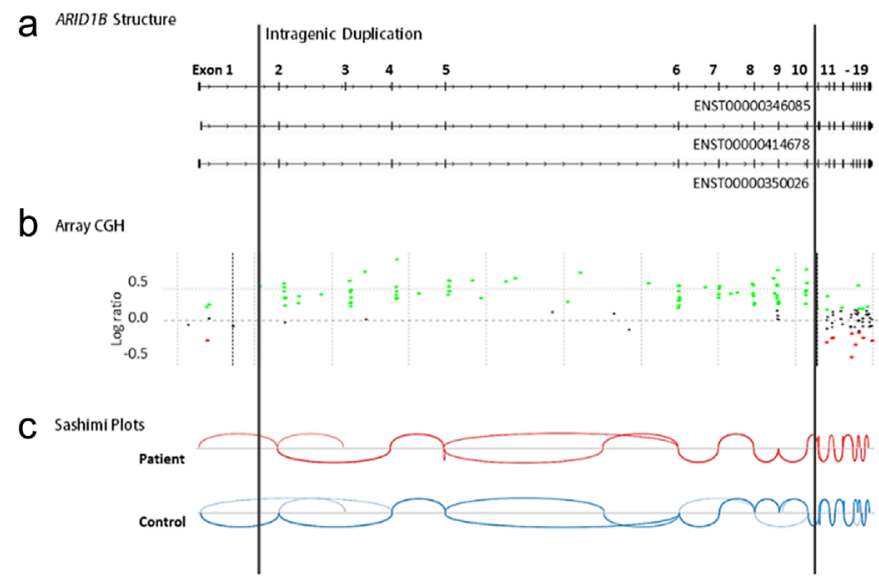

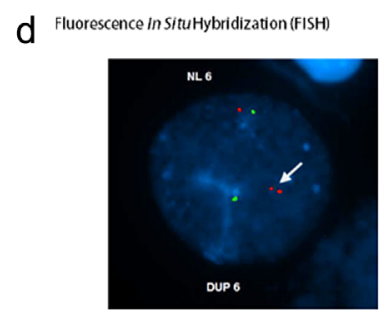

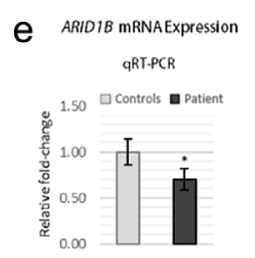

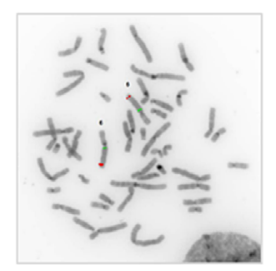
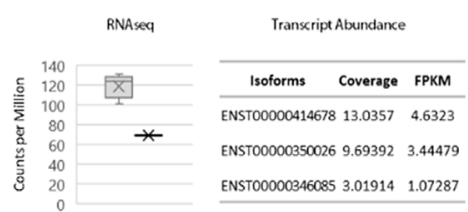

FIGURE 1 Molecular and genetic characterization of the ARID1B microduplication. (a) Representation of the ARID1B transcripts expressed in the patient with exon numbering based on an Ensembl transcript ENST00000346085. (b) Array CGH shows the duplicated region comprising exons 2 to 10 between the vertical lines. (Green dots represent probes with log ratio above 0.2 and red dots those with log ratio below -0.2. Duplication or deletion is considered when probes are $\geq 0.4$ or $\leq-0.6$, respectively.) (c) Sashimi plots generated from the RNAseq datasets depict the splice junctions that have a minimum of 3 reads supporting each junction. No novel junctions are observed in the patient. (d) FISH analysis (performed with a BAC clone, RP11-680A17, labeled in red and a control probe, RP11-719E16, labeled in green) confirms the duplication in interphase and metaphase cells, showing the duplication on chromosome 6 (as indicated by the white arrow) and not inserted into another chromosome. (e) (left to right) Decreased expression levels of ARID1B measured by qRT-PCR (using primers on exon 9 and on the junction of exons 10 and 11), in comparison to 7 age- and gender-matched controls ( $p$-val 0.02), and by RNAseq in comparison to 5 age-matched controls. Error bars represent standard deviation. Transcript abundance analysis in the patient shows the 3 ARID1B expressed transcripts, measured from the RNAseq dataset using Cufflinks. 
visualized on Integrative Genomics Viewer (Robinson et al., 2011; Thorvaldsdóttir, Robinson, \& Mesirov, 2013). Counts per Million, generated from gene level counts which were tabulated using BedTools's multibamcov algorithm (v. 2.17.0) (Quinlan \& Hall, 2010) on unique alignments for each library at all Ensembl genes (GRCh37 v.71), were calculated to compare expression levels with control samples from five healthy individuals.

\section{3 | Quantitative RT-PCR}

Quantitative RT-PCR was performed for ARID1B using custom designed primers and ACTB, GAPDH and POLR2A were used as endogenous controls. Primers were as described: ARID1B (forward tgcgtcccctcatctctcca, reverse - aggcatctgactacctggga), ACTB (forward - tgaagtgtgacgtggacatc, reverse - ggaggagcaatgatcttgat), GAPDH (forward - ggacctgacctgccgtctag, reverse - gtagcccaggatgcccttga), POLR2A (forward - gcaccacgtccaatgacat, reverse - gtgcggctgcttccataa). Primers $(0.75 \mu \mathrm{M}$ final), cDNA (1:100 final) and nuclease-free water were added to the LightCycler ${ }^{\circledR} 480$ SYBR Green I Master Mix (Roche) for a final $10 \mu \mathrm{L}$ reaction volume. A LightCycler ${ }^{\circledR} 480$ (Roche) was used for data acquisition. Values for the subject and seven age- and gendermatched controls were obtained in at least three technical replicates. Results of technical replicates for ARID1B were normalized and compared against the average of the three endogenous gene controls using the $\Delta \Delta \mathrm{Ct}$ method. Results are expressed as fold-change relative to the averaged control individuals. A two-tailed T-test was used to assess statistical significance.

\section{4 | Fluorescence in situ hybridization}

Peripheral blood from the proband was collected in a sodium heparin vacutainer tube, cultured for $72 \mathrm{hr}$ with the mitogen phytohemagglutinin, and harvested by standard cytogenetic methods. Slides containing both interphase and metaphase cells were hybridized according to standard protocol with fluorescently labeled BAC clones, RP11-680A17 and RP11-719E16, localized to 6q25.3 and $6 q 13$, respectively. The BAC clones had been grown in broth medium with $20 \mu \mathrm{g} / \mathrm{ml}$ of chloramphenicol followed by DNA extraction using a Plasmid Miniprep kit (Qiagen). The target clone, RP11-680A17, was labeled directly with Spectrum GreenTM dUTP by nick translation with the Abbott Molecular kit. After hybridization and in accordance with the laboratory's standard confirmation protocol for duplications less than $1 \mathrm{Mb}, 50$ interphase cells were manually scored using a fluorescent microscope to confirm the presence of the duplication and a metaphase cell was examined to confirm the localization of the duplicated segment.

\section{5 | Proteome analysis}

LCLs from the subject, obtained at 13 years of age, and 5 male controls ages 31-40 years were used. A total of $15 \mu \mathrm{g}$ of protein from each sample was digested with LysC for $1 \mathrm{hr}$ and trypsin overnight at $37^{\circ} \mathrm{C}$. Reverse phase LC-MS/MS was performed as described (Tomechko et al., 2015), except that $600 \mathrm{ng}$ of peptide digests was loaded on the HPLC column and the gradient of solvent B ranged from $2 \%$ to $40 \%$ over $210 \mathrm{~min}$.

Raw LC-MS/MS data files for each sample were processed using Rosetta Elucidator (Rosetta Biosoftware, Seattle, WA) (Version 3.3.01 SP4 25). Automated differential quantification of peptides was performed as previously described (Azzam et al., 2016; Neubert et al., 2008; Schlatzer, Sugalski, Dazard, Chance, \& Anthony, 2012). Briefly, LC-MS/MS raw data were imported, and for each MS spectrum profile of each LC-MS/MS run, chromatographic peaks, and monoisotopic masses were extracted and aligned. Chromatographic peaks were first aligned by retention time and monoisotopic mass. Peaklists with the monoisotopic mass and corresponding MS/MS data were then generated for each sample and searched using Mascot. Resultant peptide identifications were imported into Elucidator and monoisotopic masses annotated with peptide identifications. The false discovery rate for protein identifications was calculated to be $0.02 \%$. The MS/MS peak lists were searched by Mascot (version 2.4.1) (Matrix Science, London, UK) using the human UniProt database. Search settings were as follows: trypsin enzyme specificity; mass accuracy window for precursor ion, 25 ppm; mass accuracy window for fragment ions, 0.8 Da; variable modifications including carbamidomethlylation of cysteines, one missed cleavage and oxidation of methionine.

\subsection{Statistical methods and bioinformatic analyses}

Raw MS data were obtained for each region in a csv file; this file contained intensity values, with rows corresponding to peptides and columns corresponding to the sample. Missing values were imputed using a weighted k-nearest neighbors algorithm (Troyanskaya et al., 2001). Next, data were $\log _{2}$-transformed to achieve normality. Data were visualized with principal component analysis and complete linkage hierarchical clustering. These preprocessing steps were performed using InfernoRDN (formerly DanTE) (Polpitiya et al., 2008). Data were imported into the $R$ statistical programming environment for subsequent analyses.

Because there was one case and multiple controls, we treated individual peptides as observations of a given protein. Therefore, proteins with only one quantified peptide were excluded from our analysis. To compare protein abundance between the case and controls, we constructed a linear mixed effects model that adjusted for subject to account for the non-independence of peptides derived from the same sample. Proteins with $q<0.01$ were imported into DAVID version 6.8 (https://david.ncifcrf.gov/tools.jsp) for ontology analysis. EASE score threshold was set at a value of 1.0 and minimum count for an annotation term was set to 3 . The entire set of proteins with more than one peptide $(n=2,351)$ was used as background for enrichment analysis. Network and pathway analyses were performed in Ingenuity Pathway Analysis (IPA®, www.qiagen.com/ ingenuity); proteins with $q$-value $<0.01$ were used for this purpose. Network connections were curated based on data from all species 
and all cell lines and tissues. Enrichment scores and $p$-values for canonical pathway and network analysis were determined by a one-tailed Fisher's exact test.

\section{4 | RESULTS}

Measurement of ARID1B expression by quantitative RT-PCR showed a significant decrease of mRNA levels in the patient in comparison to control subjects ( $p$-value 0.02 ) and a similar result was observed using RNAseq (Figure 1e). Allele-specific expression could not be assessed since the subject was not heterozygous for SNPs located in coding exons or in the untranslated regions. The most abundant mRNA transcript observed in the RNAseq dataset was ENST00000414678, consistent with the GTEx database for EBV-transformed lymphoblasts; ENST00000350026 and ENST00000346085 were also detected in the patient sample (Figure 1e). IGV Sashimi plots did not reveal novel junctions or transcripts for this gene (Figure 1c), providing no evidence that duplication of this exonic region resulted in alterations in splicing.

Follow-up fluorescence in situ hybridization analyses showed the presence of duplicated ARID1B genome in interphase cells (Figure 1d). The latter, in turn, was localized to chromosome 6q25.3 on analysis of a metaphase cell, the site of the ARID1B gene (Figure 1d), confirming that the microduplication occurred within the same chromosome and excluding the hypothesis of complex rearrangements.

Proteomic analyses of the LCLs resulted in the quantification of 15,792 peptides, corresponding to 3,426 proteins. There were 1,014 proteins that were differentially expressed between the case and controls $(q<0.01)$. The differentially expressed proteins and related data are noted in Supplementary Table S1. Principal component analysis and hierarchical clustering analysis revealed marked separation of the subject's proteome from that of controls (Supplementary Figure S1).

Gene ontology analysis in DAVID revealed enrichment of several annotation clusters with terms relating to ATP binding, mitochondrion, and flavin adenine dinucleotide (Supplementary Table S2). Bioinformatic analysis with IPA $®$ revealed enrichment for canonical pathways of EIF2 signaling ( $p$-value 6.31 E-17), protein ubiquitination ( $p$-value $1.58 \mathrm{E}-16$ ), regulation of elF4 and p70S6 K signaling ( $p$-value $1.00 \mathrm{E}-14)$, tRNA charging ( $p$-value $5.01 \mathrm{E}-13$ ), and cell cycle control of chromosomal replication ( $p$-value $6.31 \mathrm{E}$-11) (Supplementary Table S3). Downregulation of: (1) intracellular components involved in intracellular organization of membranes, organelles, and vesicles; (2) aspects of cell cycle control, signal transduction, and nuclear protein export; (3) ubiquitination and proteosomal function; and (4) aspects of mRNA synthesis/splicing are noted in IPA ${ }^{\circledR}$ network analyses (Supplementary Figures S2a-d).

\section{5 | DISCUSSION}

The subject has a unique ARID1B mutation, a copy number gain involving part of that gene that was initially determined by a chromosomal microarray analysis and which was thought likely to cause a pathologic reduction of ARID1B gene expression. Subsequent gene expression data, by both qRT-PCR and RNAseq, indicated haploinsufficiency of ARID1B and was consistent with localization of the duplication within the ARID1B gene, disrupting expression of the affected allele. Confirmation that there is a large intragenic ARID1B duplication was established by a fluorescence in situ hybridization analysis. Haploinsufficiency for ARID1B is likely to be the cause of developmental delay in this individual as no additional genetic or metabolic defects were identified. While the measurement of RNA in LCLs does not necessarily reflect the effect of the genetic lesion in the brain, the association of heterozygous inactivating mutations in ARID1B with neurodevelopmental phenotypes in other subjects suggests that reduced gene expression also occurs in the central nervous system. As $A R I D 1 B$ is expressed at different levels in the brain, namely higher in the cerebellum than in many peripheral tissues and other measured areas of the brain (http://www.gtexportal.org/), the consequences of its reduced expression may be even more pronounced there, possibly accounting for aspects of subject's phenotype.

There are two reports of smaller microduplications of ARID1B. The clinical and genetic phenotypes of these two individuals and our case are summarized in Supplementary Table S4. Our case has several unique aspects to his phenotype. Few individuals with $A R I B 1 B$ haploinsufficiency are reported with low-normal intellectual function (Santen \& Clayton-Smith, 2014). Our patient, while having a significant speech/language disorder and cognitive disability, has had several IQ determinations within the normal range but there was considerable (and reproducible) variability in selected skills. In addition, his clinical course has been characterized by easy fatigability and episodes of developmental regression, prompting evaluation for a possible metabolic underpinning of these clinical concerns. Apart from intermittent elevated blood lactate levels, extensive metabolic testing was unrevealing. To our knowledge, other cases of individuals with ARID1B haploinsufficiency do not have histories of episodic regression, although the episodic memory dysfunction in one case may be relevant (Yu et al., 2015).

Our results support the view that $A R I D 1 B$ is a dosage sensitive gene whose expression can be affected by deletions or duplications. Indeed, the evolutionary constraint on this gene shows that it is highly intolerant to loss-of-function mutations according to its ExAC pLI score of 1.00 (http://exac. broadinstitute.org/). As we did not identify novel transcripts, the duplicated allele either was not transcribed or was transcribed but its RNA was degraded by surveillance mechanisms. Other chromatin regulators have also been noted to be dosage sensitive causes of neurodevelopmental phenotypes, including MBD5, EHMT1, CHD8, and SATB2 (Talkowski et al., 2012).

The lymphoblast proteomic data are striking: statistically highly significant dysregulation of pathways related to initiation of translation were noted, in addition to dysregulation of pathways 
and networks relating to protein ubiquitination, mTOR signaling, apoptosis, major metabolic pathways (e.g., TCA cycle, mitochondrial oxidative phosphorylation), cell cycle control, mRNA synthesis/processing, and intracellular vesicular transport. A number of the differentially expressed pathways and networks relate to described roles of ARID1B and the SWI/SNF-A chromatin remodeling complex in different tissues such as cell cycle regulation, ubiquitination, mRNA synthesis/processing, apoptosis, and intracellular signaling pathways ( $\mathrm{Li}$, Trojer, Matsumura, Treisman, \& Tanese, 2010; Nagl, Wang, Patsialou, Van Scoy, \& Moran, 2007; Raab, Resnick, \& Magnuson, 2015; Sim et al., 2015; Xi, He, Zhang, Le, \& Massagué, 2008); involvement of other pathways, including many metabolic pathways and intracellular vesicular trafficking, is newly described. Our data do not specifically implicate dysregulation of $\mathrm{Wnt} / \beta$-catenin signaling as was noted in another recent study (Vasileiou et al., 2015). Yet, although our proteomic analysis indicates widespread and marked differential expression of proteins in the ARID1B haploinsufficient lymphoblasts, there are three important potential limitations of these findings: first, one cannot generalize these results until proteomic analysis of additional cases of ARID1B haploinsufficiency are carried out; second, the proteomic findings, while predictive of marked dysregulation of multiple pathways and networks, do not reveal which dysregulated pathways are of greatest pathophysiological significance; and, third, the relevance of lymphoblast findings to brain biology (and that of other tissues) is uncertain.

The roles of ARID1B in brain development are starting to be understood. Recent studies reveal that it may be critical in cell proliferation and differentiation and is important in dendritic arborization, and synapse formation (Harmacek et al., 2014; Nagl et al., 2007; Tuoc et al., 2013; Yan et al., 2008). Consequently, ARID1B deficiency may result in neurodevelopmental defects via abnormal brain wiring induced by the defective differentiation of neurons (Ka, Chopra, Dravid, \& Kim, 2016). This report provides additional perspectives regarding ARID1B haploinsufficiency and its associated developmental disabilities. Further studies are needed to understand the precise mechanisms whereby altered chromatin remodeling leads to changed brain development and cognitive function.

\section{ACKNOWLEDGMENTS}

We thank the family for participating in this case report. Funding was provided by FCT Fellowship SFRH/BD/52049/2012 to CMS NIH grant GM061354 to JFG, and MET, SFARI grant 308955 to JFG and R0OMH095867 to MET and Autism Research Institute grant to MRN.

\section{CONFLICTS OF INTEREST}

The authors have no conflicts of interest regarding this work.

\section{REFERENCES}

American Psychiatric Association. (2013). Diagnostic and Statistical Manual of Mental Disorders (DSM-5) (5th Ed.). Washington, DC: American Psychiatric Association.

Azzam, S., Schlatzer, D., Maxwell, S., Li, X., Bazdar, D., Chen, Y., .. Sieg, S. F. (2016). Proteome and protein network analyses of memory $T$ cells find altered translation and cell stress signaling in treated human immunodeficiency virus patients exhibiting poor CD4 recovery. Open Forum Infectious Diseases, 3, ofw037.

Cheung, S. W., Shaw, C. A., Yu, W., Li, J., Ou, Z., Patel, A., .. Beaudet, A. L. (2005). Development and validation of a CHG microarray for clinical cytogenetic diagnosis. Genetics in Medicine, 7, 422-432.

DeLuca, D. S., Levin, J. Z., Sivachenko, A., Fennell, T., Nazaire, M.-D., Williams, C., ... Getz, G. (2012). RNA-SeQC: RNA-seq metrics for quality control and process optimization. Bioinformatics, 28, 1530-1532.

Euskirchen, G., Auerbach, R. K., \& Snyder, M. (2012). SWI/SNF chromatinremodeling factors: Multiscale analyses and diverse fnctions. Journal of Biological Chemistry, 287, 30897-30905.

Harmacek, L., Watkins-Chow, D. E., Chen, J., Jones, K. L., Pavan, W. J., Salbaum, J. M., \& Niswander, L. (2014). A unique missense allele of BAF155, a core BAF chromatin remodeling complex protein, causes neural tube closure defects in mice. Developmental Neurobiology, 74, 483-497.

Hoyer, J., Ekici, A. B., Endele, S., Popp, B., Zweier, C., Wiesener, A., . . Reis, A. (2012). Haploinsufficiency of ARID1B, a member of the SWI/SNF-A chromatin-remodeling complex, is a frequent cause of intellectual disability. American Journal of Human Genetics, 90, 565-572.

Ka, M., Chopra, D. A., Dravid, S. M., \& Kim, W.-Y. (2016). Essential roles for ARID1B in dendritic arborization and spine morphology of developing pyramidal neurons. Journal of Neuroscience, 36 , 2723-2742.

Li, H., Handsaker, B., Wysoker, A., Fennell, T., Ruan, J., Homer, N., ... Durbin, R. (2009). The sequence alignment/map format and SAM tools. Bioinformatics, 25, 2078-2079.

Li, X. S., Trojer, P., Matsumura, T., Treisman, J. E., \& Tanese, N. (2010). Mammalian SWI/SNF-A subunit BAF250/ARID1 is an E3 ubiquitin ligase that targets histone H2B. Molecular and Cellular Biology, 30, 1673-1688.

Lu, X., Shaw, C. A., Patel, A., Li, J., Cooper, M. L., Wells, W. R., ... Ward, P. A. (2007). Clinical implementation of chromosomal microarray analysis: Summary of 2513 postnatal cases. PLoS ONE, 2, e327.

Nagl, N. G., Wang, X., Patsialou, A., Van Scoy, M., \& Moran, E. (2007). Distinct mammalian SWI/SNF chromatin remodeling complexes with opposing roles in cell-cycle control. EMBO Journal, 26, 752-763.

Neubert, H., Bonnert, T. P., Rumpel, K., Hunt, B. T., Henle, E. S., \& James, I. T. (2008). Label-free detection of differential protein expression by LC/MALDI mass spectrometry. Journal of Proteome Research, 7, 2270-2279.

Polpitiya, A. D., Qian, W., Jaitly, N., Petyuk, V. A., Adkins, J. N., Camp, D. G. II, ... Smith, R. D. (2008). DAnTE: A statistical tool for quantitative analysis of -omics data. Bioinformatics, 24, 1556-1558.

Quinlan, A. R., Hall, I. M. (2010). BEDTools: A flexible suite of utilities for comparing genomic features. Bioinformatics, 26, 841-842.

Raab, J. R., Resnick, S., \& Magnuson, T. (2015). Genome-wide transcriptional regulation mediated by biochemically distinct SWI/SNF complexes. PLoS Genetics 11:e 1005748.

Robinson, J. T., Thorvaldsdóttir, H., Winckler, W., Guttman, M., Lander, E. S., Getz, G., \& Mesirov, J. P. (2011). Integrative genomics viewer. Nature Biotechnology, 29, 24-26.

Santen, G. W. E., Clayton-Smith, J. (2014). The ARID1B phenotype: What we have learned so far. American Journal of Medical Genetics Part C, 166C, 276-289. 
Schlatzer, D. M., Sugalski, J., Dazard, J.-E., Chance, M. R., \& Anthony, D. D. (2012). A quantitative proteomic approach for detecting protein profiles of activated human myeloid dendritic cells. Journal of Immunological Methods, 375, 39-45.

Sim, J. C. H., White, S. M., \& Lockhart, P. J. (2015). ARID1B-mediated disorders: Mutations and possible mechanisms. Intractable \& Rare Diseases Research, 4, 17-23.

Talkowski, M. E., Rosenfeld, J., Blumenthal, I., Pillalamarri, V., Chiang, C., Heilbut, A., ... Gusella, J. F. (2012). Sequencing chromosomal abnormalities reveals neurodevelopmental loci that confer risk across diagnostic boundaries. Cell, 149, 525-537.

Thorvaldsdóttir, H., Robinson, J. T., \& Mesirov, J. P. (2013). Integrative genomics viewer (IGV): High-performance genomics data visualization and exploration. Briefings in Bioinformatics, 14, 178-192.

Tomechko, S. E., Lundberg, K. C., Jarvela, J., Bebek, G., Chesnokov, N. G., Schlatzer, D., ... Silver, R. F. (2015). Proteomic and bioinformatics profile of paired human alveolar macrophages and peripheral blood monocytes. Proteomics, 15, 3797-3805.

Troyanskaya, O., Cantor, M., Sherlock, G., Brown, P., Hastie, T., Tibshirani, R., ... Altman, R. B. (2001). Missing value estimation methods for DNA microarrays. Bioinformatics, 17, 520-525.

Tuoc, T. C., Boretius, S., Sansom, S. N., Pitulescu, M.-E., Frahm, J., Livesey, F. J., \& Stoykova, A. (2013). Chromatin regulation by BAF170 controls cerebral cortical size and thickness. Developmental Cell, 25, 256-269.

Vasileiou, G., Ekici, A. B., Uebe, S., Zweier, C., Hoyer, J., Engels, H., ... Hadjihannas, M. V. (2015). Chromatin remodeling factor ARID1B represses Wnt/ $\beta$-catenin signaling. American Journal of Human Genetics, 97, 445-456.

Wang, L., Wang, S., \& Li, W. (2012). RSeQC: Quality control of RNA-seq experiments. Bioinformatics, 28, 2184-2185.
Wu, T. D., Nacu, S. (2010). Fast and SNP-tolerant detection of complex variants and splicing in short reads. Bioinformatics, 26 , 873-881.

Xi, Q., He, W., Zhang, X. H., Le, H. V., \& Massagué, J. (2008). Genome-wide impact of the BRG1 SWI/SNF chromatin remodeler on the transforming growth factor $\beta$ transcriptional program. Journal of Biological Chemistry, 283, 1146-1155.

Yan, Z., Wang, Z., Sharova, L., Sharov, A. A., Ling, C., Piao, Y., ... Ko, M. S. H. (2008). BAF250B-associated SWI/SNF chromatin-remodeling complex is required to maintain undifferentiated mouse embryonic stem cells. Stem Cells, 26, 1155-1165.

Yu Y, Yao R, Wang L, Fan Y, Huang X, Hirschhorn J, ... Shen Y. 2015. De novo mutations in ARID1B associated with both syndromic and non-syndromic short stature. BMC Genomics 16:701.

\section{SUPPORTING INFORMATION}

Additional Supporting Information may be found online in the supporting information tab for this article.

How to cite this article: Seabra CM, Szoko N, Erdin S, et al. A novel microduplication of ARID1B: Clinical, genetic, and proteomic findings. Am J Med Genet Part A. 2017;1-7.

https://doi.org/10.1002/ajmg.a.38327 